\title{
Mitigation of Nonlinear Effects on WDM QAM Signals Enabled by Optical Phase Conjugation with Efficient Bandwidth Utilization
}

\author{
Satoshi Yoshima, Yujia Sun, Zhixin Liu, Member, IEEE, Kyle R. H. Bottrill, Francesca Parmigiani, \\ David J. Richardson, Fellow, IEEE, and Periklis Petropoulos
}

\begin{abstract}
We present nonlinear impairment mitigation of wavelength division multiplexed (WDM) signals, through optical phase conjugation (OPC). We conduct our experiments on a 400-km long installed fiber link equipped with erbium-doped fiber amplifiers (EDFAs), with the OPC placed close to the middle of the link. Our OPC configuration realizes efficient reuse of the signal bandwidth, avoiding the loss of half of the spectral band typical of most phase conjugating schemes. We demonstrate the operation of the system using both 16- and 64- quadrature amplitude modulation (QAM) signals and report Q-factor improvements up to 0.5 and $2.5 \mathrm{~dB}$ for 16- and 64-QAM, respectively.
\end{abstract}

Index Terms - Fiber nonlinearity, nonlinear noise mitigation, optical fiber communication, optical phase conjugation, wavelength division multiplexing.

\section{INTRODUCTION}

A DVANCED modulation formats, such as quadrature amplitude modulation (QAM), where information is imprinted on both the amplitude and the phase of the transmitted signals, offer a route towards efficient use of the transmission spectrum and, thus, are promising candidates to meet the demand for high capacity in future long haul optical transmission systems [1, 2]. However, to achieve similar levels of data transmission fidelity as simpler formats, they generally require higher launch powers for the same transmission length, due to their requirement for a higher optical signal to noise ratio (OSNR) at the receiver. This is challenging when transmitting signals over long fiber lengths, where Kerr nonlinearity limits the maximum signal power that can be launched into the fiber

Manuscript received June xx, 2016. This experiment was performed on the jointly EPSRC/JISC supported NDFIS Aurora2. The work is part of the EPSRC Photonics Hyperhighway Programme Grant. The data for this work is accessible through the University of Southampton Institutional Research Repository (http://dx.doi.org/10.5258/SOTON/396182)

S. Yoshima is with the information Technology R\&D Center, Mitsubishi Electric Corporation, Kanagawa, 247-8501, Japan (e-mail: Yoshima.Satoshi@aj.MitsubishiElectric.co.jp). He was with the Optoelectronics Research Centre, University of Southampton, Southampton, SO17 1BJ, U.K.

Y. Sun, Z. Liu, K. R. H. Bottrill, F. Parmigiani, D. J. Richardson, and P. Petropoulos are with the Optoelectronics Research Centre, University of Southampton, Southampton, SO17 1BJ, U.K. (e-mail: ys9g11@soton.ac.uk; z.liu@soton.ac.uk; k.bottrill@soton.ac.uk; frp@orc.soton.ac.uk; djr@orc.soton.ac.uk; pp@orc.soton.ac.uk). link, thereby compromising system performance [3]. Several approaches have been investigated, in both the digital and optical domains, in order to compensate for deterministic nonlinear impairments. They include: digital back-propagation [4], phase sensitive amplification [5-7], phase-conjugated twin waves [8], multiple OPCs [9, 24], and mid-link OPC [11-18]. Amongst these, mid-link OPC, located at exactly the middle point of the transmission link has regained some momentum in recent years due to the exceptional performance it allows for a relatively simple modification of the overall system. More importantly, OPC is modulation-format transparent, can handle high speed and heterogeneous signals, and is capable of concurrently operating upon an entire WDM band. Furthermore, unlike digital techniques, its benefits usually come at less additional cost to latency, since the compensation occurs during propagation in the transmission fiber itself [19]. Similarly, the use of multiple OPCs, where a number of OPC units are used instead, each covering a shorter transmission span, is attractive and can in principle achieve better performance than mid-link OPC in terms of overall nonlinear compensation [13]. In this instance, symmetric loss and dispersion profile can be more easily realized and, more importantly, the states of polarization among signals can be more fairly maintained, ensuring that the corresponding nonlinear phase noise accumulated prior to the OPC can be undone through transmission after the OPC [13]. A performance study of the cascadability of multiple OPCs has been carried out in [9]. From a practical viewpoint, however, it might be difficult to set OPC units at several points of an existing transmission line where the loss profile has already been optimized for operation with the existing EDFA technology.

As twice the transmission bandwidth is required during the process of optical phase conjugation, typical OPC demonstrations either make use of two nonlinear processing media, conjugating half the band in each [13], or otherwise only occupy half of the WDM band during transmission, reserving the rest for the conjugated idlers produced during OPC. Therefore, OPC is usually associated with inefficient use of spectral resources. Moreover, the majority of experimental demonstrations to date have been carried out in laboratory environments and exploit system designs that allow for nearly ideal nonlinear compensation, using for example distributed 
Raman amplification to symmetrize the power evolution about the OPC, and/or properly designed transmission fibers to satisfy both second- and third-order dispersion requirements [9-14]. This is especially the case when more advanced modulation format signals are transmitted.

With respect to more complex modulation formats, K. Solis-Trapala et al. [17] demonstrated the doubling of the transmission reach for a single dual-polarization 64-QAM signal over a field-deployed legacy fiber system using mid-link OPC. However, as previously highlighted, one of the most important benefits of OPC is the capability to process a number of WDM channels simultaneously. We have recently reported the transmission of six 64-QAM signals in an OPC-based system arranged in two WDM sub-bands [21]. However, the characterization presented in that report was incomplete and concerned only two of the transmitted channels.

In this paper, expanding from the work we presented in [20, 21], we experimentally demonstrate fiber nonlinearity mitigation enabled by OPC, located close to the middle point (at a location dictated by practical access considerations) of a field installed, amplified, standard single mode fiber (SMF) link with a total length of about $400 \mathrm{~km}$ for two different modulation formats, 16- and 64- QAM. In order to avoid reserving a band for wavelength conversion as well as for economy in components, a counter-propagating scheme was used in a single highly nonlinear fiber (HNLF) employing the same two pumps in both directions [22], while polarization insensitivity was guaranteed using orthogonally polarized pumps along each propagation direction [23]. We studied the system performance in terms of signal quality factor (Q-factor) and bit error ratio (BER) with and without OPC, and the effect of increasing the number of signals present during the transmission, clearly highlighting the enhancement in performance when OPC was employed, especially for signals with high OSNR requirements. We present full characterization of the performance of six channels arranged in two WDM sub-bands. We also discuss the benefits offered by our OPC scheme in terms of efficient bandwidth utilization and compare it with other approaches.

\section{EXPERIMENTAL SET-UP}

Figure 1 compares the bandwidth utilization of conventional OPC schemes to our proposed method. As shown in Fig. 1(a), conventional OPC configurations usually occupy only half of the WDM band during transmission, reserving the other half to the idlers generated during the phase conjugating process. To take advantage of the entire transmission bandwidth, a few solutions have been proposed [13-14, 20-21]. They are all

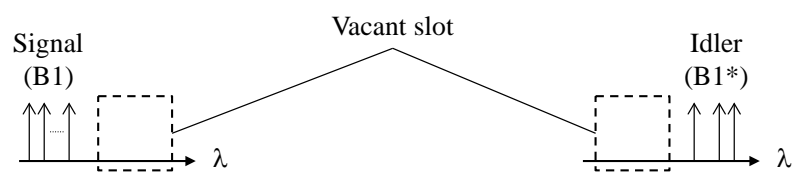

Before OPC

After OPC

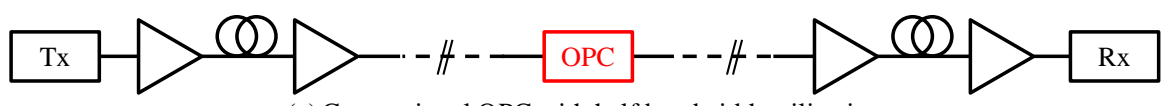

(a) Conventional OPC with half bandwidth utilization
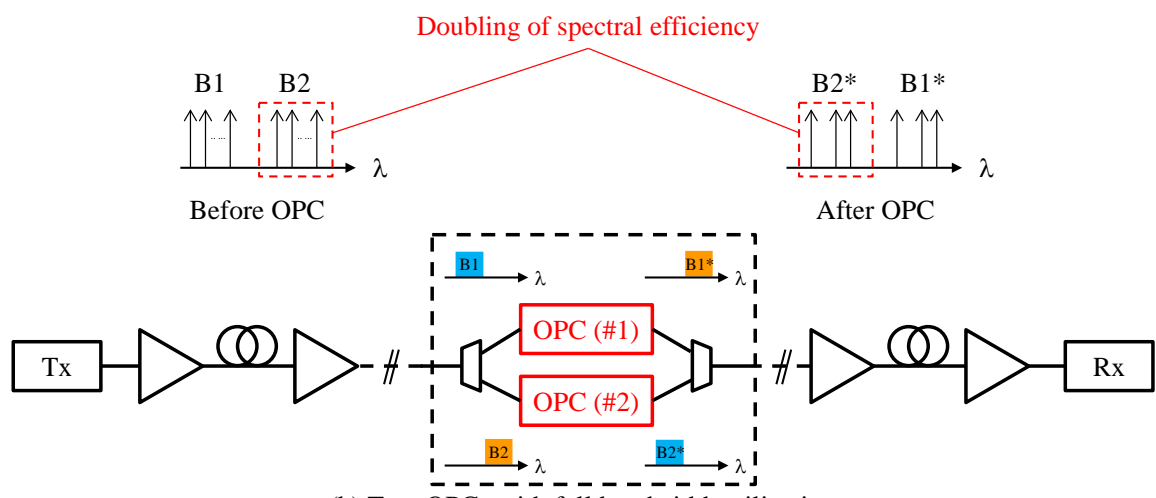

(b) Two OPCs with full bandwidth utilization

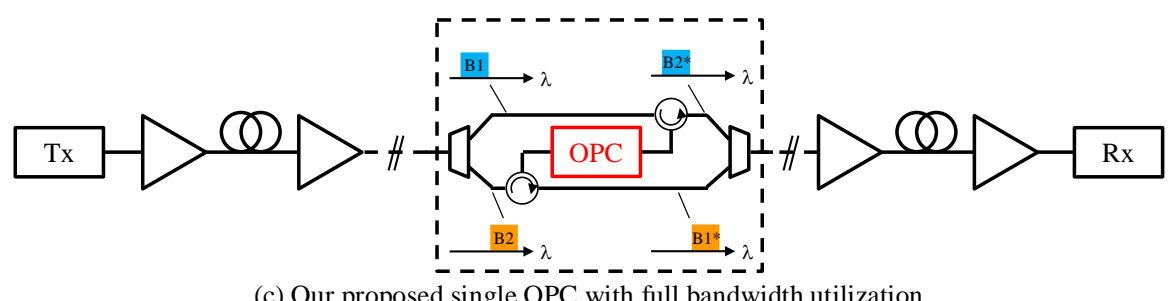

(c) Our proposed single OPC with full bandwidth utilization

Fig. 1. Comparison of OPC approaches in terms of both efficient bandwidth utilization and number of OPCs. 


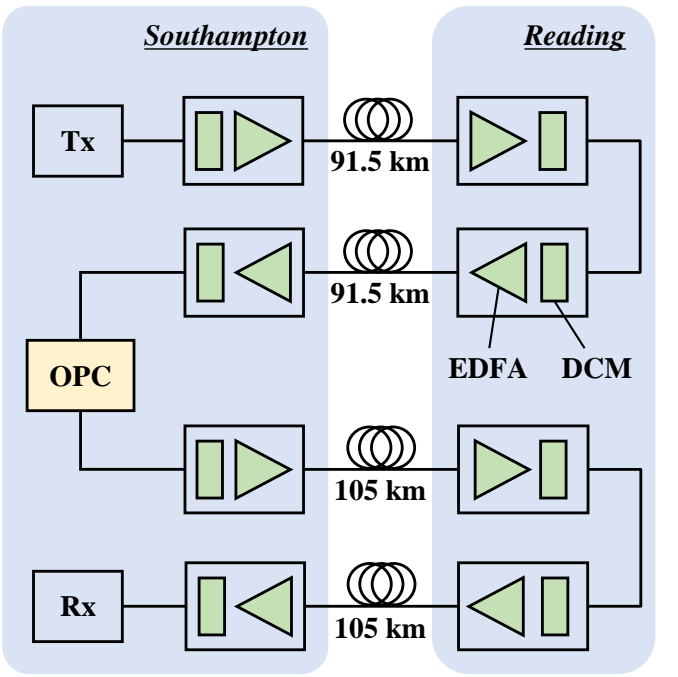

(a) Field trial setup

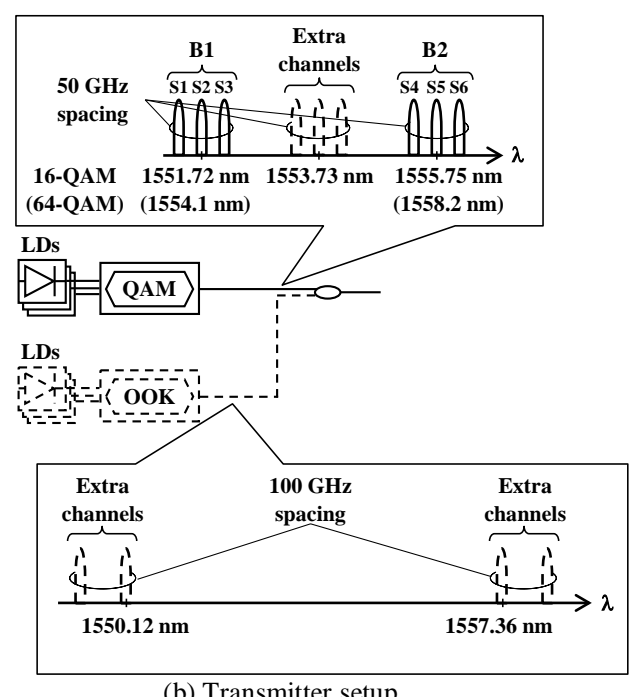

(b) Transmitter setup

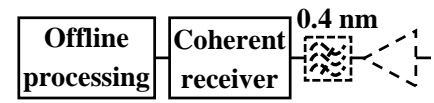

(c) Receiver setup

Fig. 2. Experimental setup: Field trial (a), transmitter (b) and receiver (c) set-up.

based on the assumption that the entire bandwidth is divided into two sub-bands and each sub-band is processed by an independent optical phase conjugator. In [13-14] signal polarization insensitivity of the phase conjugator was achieved using polarization diversity applied either in a HNLF [13] or in a periodically poled lithium niobate (PPLN) waveguide [14], where each signal polarization propagated in an opposite direction of the same nonlinear medium via a Sagnac loop [25]. At least two different nonlinear media needed to be used to allow processing of the two sub-bands, which we call B1 and B2 in Fig. 1. In our proposed scheme $[20,21]$ signal polarization insensitivity is guaranteed by using two orthogonally polarized pumps in the phase conjugation process and a low birefringence nonlinear medium (a HNLF in our case) [26]. As only a single pass in the nonlinear medium is necessary, the counter-propagating direction can be used for the second sub-band, see Fig. 1(c), thus halving the number of required nonlinear devices as compared to the previous schemes.

Figure 2(a) shows the experimental setup of the installed transmission link (part of the UK’s Aurora2 network) including the OPC, set approximately at the middle point of the transmission line. The transmitter (Tx), OPC, and receiver (Rx) were located at Southampton, while the repeaters, including EDFAs and dispersion compensation modules (DCMs), were located at both Southampton and Reading. The fully compensated (both in terms of dispersion and dispersion slope) field-installed fiber was standard SMF (ITU-T G.652d). The total transmission length of about $400 \mathrm{~km}$ consisted of two round trips with the OPC sat in-between; the first, about $183 \mathrm{~km}$ long, and the second, about $210 \mathrm{~km}$ long. Each DCM compensated for approximately half of the dispersion of the span length of SMF that followed/preceded it. The asymmetry in the lengths of the two halves of the line was due to added fiber spools of standard SMFs in the second half of the transmission link (positioned in the laboratory at Southampton). In more detail, we added about $1 \mathrm{~km}$ of SMF in the first span and about $28 \mathrm{~km}$ in the second one. These lengths of extra
SMFs were selected to maximize the measured Q-factors of received signals after transmission in each round trip separately (when OPC was not included). This was done to allow for a more accurate dispersion compensation in each span with the dispersion compensation modules we had available. As a result, the second round trip was about $27 \mathrm{~km}$ longer than the first one; this situation is typical in many real-world deployed networks where it might not be possible to secure presence exactly at the mid-point of the transmission. It is worth noting that while the performance of OPC-based systems is optimized under conditions of a symmetric transmission line and power profile [9], significant mitigation of nonlinearities can still be achieved at non-optimum conditions, as we will demonstrate herein.

Figure 2(b) shows the experimental setup of the transmitter. The two signal bands, B1 and B2, were centered at $1551.72 \mathrm{~nm}$ $(1554.1 \mathrm{~nm})$ and $1555.75 \mathrm{~nm}(1558.2 \mathrm{~nm})$, respectively, for 16-QAM (64-QAM) and each contained three 10 Gbaud QAM signals lying on a $50 \mathrm{GHz}$ grid. For convenience, we will call the three B1 channels S1-S3, and the B2 channels S4-S6. In order to study the system performance for an increased amount of nonlinear impairments, for the 16-QAM case, we also conducted experiments while an additional number of channels were present during the transmission. In particular, a third QAM sub-band was added, centered around $1553.73 \mathrm{~nm}$, along with four 10 Gbaud on-off keying (OOK) signals positioned at $1549.32 \mathrm{~nm}, 1550.12 \mathrm{~nm}, 1557.36 \mathrm{~nm}$, and $1558.17 \mathrm{~nm}$, respectively.

At the OPC point, the signals were input to a wavelength selective switch (WSS) and directed at different output ports. The demultiplexed B1 and B2 sub-bands were directed to the OPC inputs, while all remaining signals (when present) by-passed the OPC and were launched in to the second part of the transmission link. When transmission was evaluated without the OPC for comparative purposes, the incoming signals were all directed to the by-pass port. Figure 3 shows the implementation of the polarization insensitive OPC based on two orthogonal pumps [23]. The wavelengths of the two CW pumps were $1558.17 \mathrm{~nm}(1560.6 \mathrm{~nm})$ and $1549.32 \mathrm{~nm}$ (1551.7 


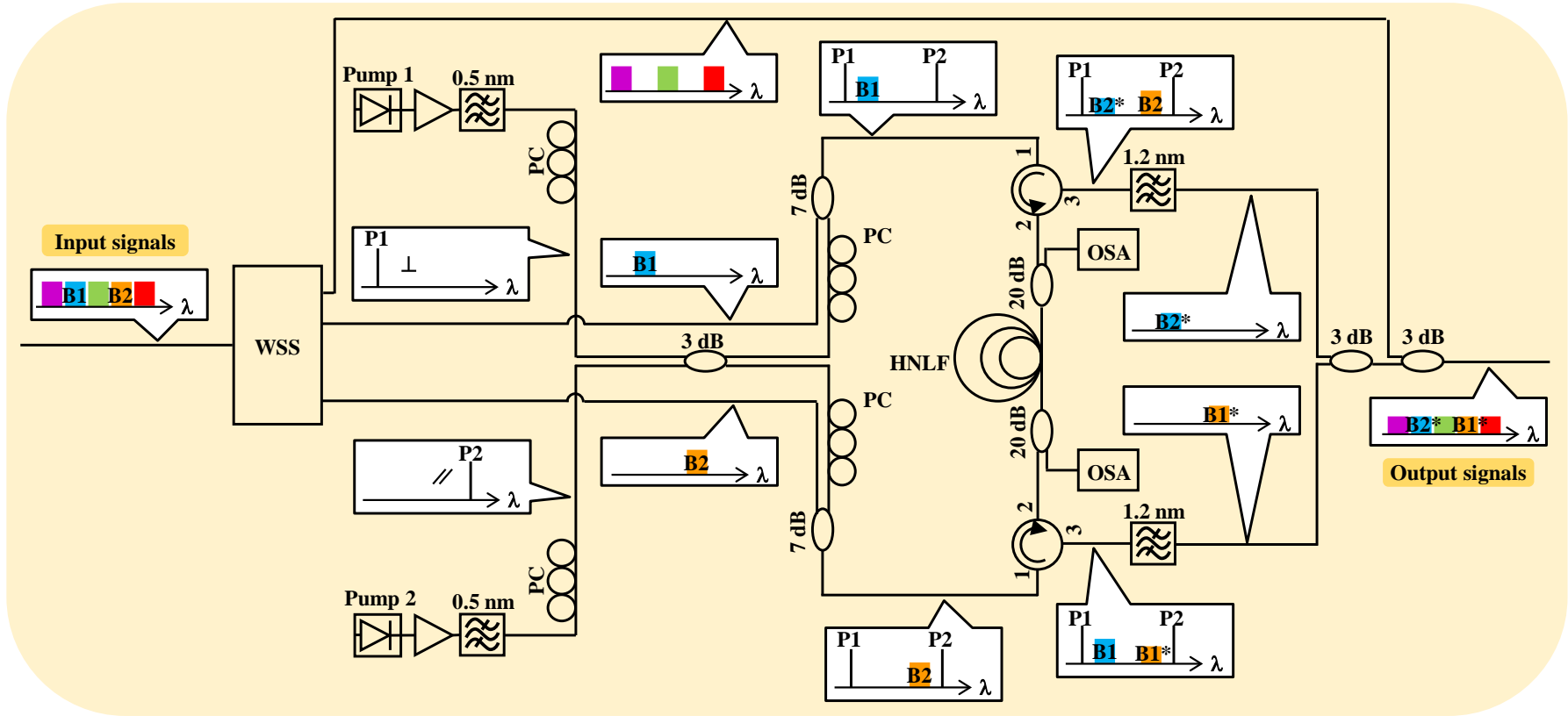

Fig. 3. OPC schematic.

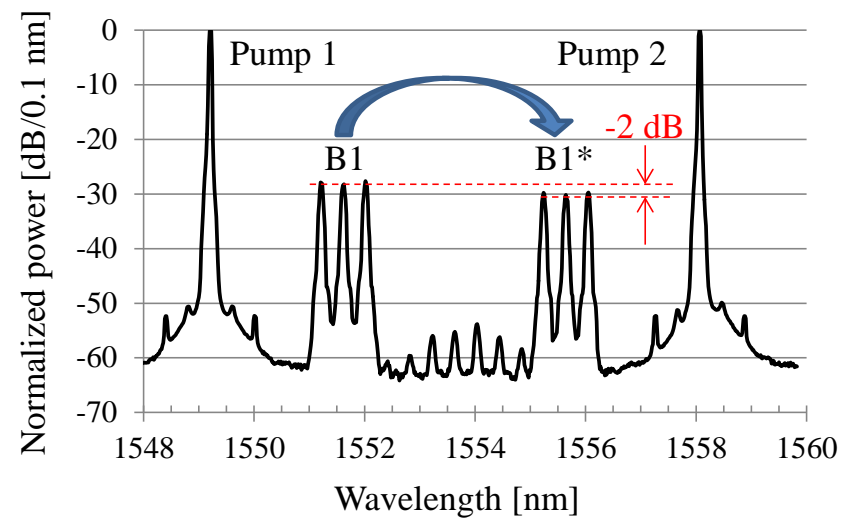

(b) From B1 to B1*

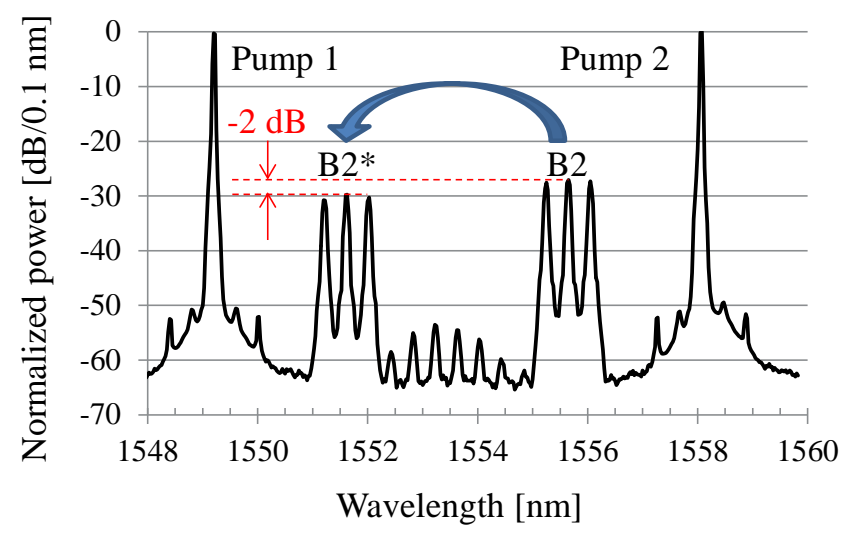

(b) From B2 to B2*

Fig. 4. Typical spectra ( $0.1 \mathrm{~nm}$ resolution) at the output of the HNLF for B1 and B2 (both with a conversion efficiency of $-2 \mathrm{~dB}$ ).

$\mathrm{nm}$ ) for 16-QAM (64-QAM) and each had a linewidth of about $100 \mathrm{kHz}$. The pump sources were amplified separately to ensure that the power of each one was $+22.0 \mathrm{dBm}$ at each of the two HNLF inputs. Optical band-pass filters (OBPFs) with a full-width at half maximum (FWHM) of $0.5 \mathrm{~nm}$ were used to suppress amplified spontaneous emission (ASE) noise from the EDFAs. Two polarization controllers (PCs) following the OBPFs were used to guarantee the orthogonality of the pumps. After combining the two pumps and directing them to both directions of the HNLF, B1 and B2 were also separately combined with each pump pair using a $7 \mathrm{~dB}$ coupler and launched via circulators into the different propagation directions of the fiber. The 300-m long germanium-doped strained HNLF had a nonlinear coefficient of $11.6 /(\mathrm{W}-\mathrm{km})$, a dispersion of $-0.08 \mathrm{ps} /(\mathrm{nm}-\mathrm{km})$, a dispersion slope of 0.018 $\mathrm{ps} /\left(\mathrm{nm}^{2}-\mathrm{km}\right)$, and a zero-dispersion wavelength of $1555 \mathrm{~nm}$. The stimulated Brillouin scattering (SBS) threshold of the HNLF was $+24 \mathrm{dBm}$, i.e. higher than the pump power used. The conversion efficiency of the OPC was the same in both directions and was about $-2 \mathrm{~dB}$ uniformly across the signal bands. Typical spectra obtained at the two opposite HNLF outputs are shown in Fig. 4(a) and (b). Even though the pump polarizations were adjusted manually in this experiment, it has been shown in [23] that a feedback circuit that monitors the nonlinear interaction between the two pumps can automatically adjust their states of polarization, so that they remain orthogonal to each other. The implementation of the feedback circuit is based on commercially available polarization trackers. In our case, we found that manual control allowed us to keep the polarization of the two pumps stable over a period of a few tens of minutes.

To avoid the sacrifice of any additional spectral bands while carrying out OPC, the conjugated B1 sub-band (which we call B1*) occupied the wavelengths of B2 and vice versa. The generated idlers (B1* and $\mathrm{B} 2 *$ ) were selected using tunable filters with FWHM of $1.2 \mathrm{~nm}$ which removed the corresponding signal sub-bands (B1 and B2) as well as the pumps and were combined together at the input of the second half of the transmission link, as depicted in Fig. 3. If other signals were present they were also combined with the B1* and 
B2* signal sub-bands before further transmission.

At the receiver, the signal under test was filtered and detected by a coherent receiver and a real time scope, using a commercial optical modulation analyzer (OMA) to evaluate its Q-factor and the corresponding BER values.

We carried out two sets of experiments, one with 16-QAM and another with 64-QAM signals, and these experiments are described below.

\section{TRANSMISSION EXPERIMENT FOR 16-QAM WDM SIGNALS}

In the first set of experiments, we studied nonlinear impairment mitigation of 16-QAM WDM signals. We initially investigated the transmission performance with and without OPC, when only the two B1 and B2 sub-bands were transmitted. Figure 5 shows the Q-factors of the middle channels in each sub-band (S2 and S5, respectively) after $400 \mathrm{~km}$ transmission, as a function of launched power per channel in each fiber span. Both Q-factors of I2 and I5 (the respective conjugates of S2 and S5) were superior to the case without OPC for all launch powers, demonstrating a Q-factor improvement of $0.5 \mathrm{~dB}$ for both idlers and a $1 \mathrm{~dB}$ higher optimum launched power per channel with OPC than without OPC due to the fiber Kerr nonlinearity mitigation. Note that the repeatability of these results was confirmed to within $+/-0.1 \mathrm{~dB}$ for all channels. The relatively small improvement may be due to the negative conversion efficiency in the OPC, non-optimum symmetric transmission length, power profile and relatively short transmission lengths. Note that the slight difference of about $0.2 \mathrm{~dB}$ in the Q-factor measurements between the two signals (S2 and S5 and I2 and I5) was due to the variation in quality of the signals at the transmitter and the slightly different optical signal to noise ratios of the WDM signals due to the non-uniform amplification gain in the transmission link. The constellation maps with and without OPC at a launched power of $0 \mathrm{dBm}$ for B1 band (S2 and I2) are also reported in Fig. 5. The reduction in phase noise through the use of OPC is visible for all symbols, but is especially noticeable at the outer symbols of the constellation (i.e. those bearing a higher power). Similar Q-factor improvements were measured for all remaining channels as shown in the top of Fig. 6 (triangle symbols). It is interesting to note that the middle channels in each sub-band (S2 and S5), which experience the most nonlinearity during propagation, are the ones showing the highest level of improvement. In addition, we also investigated the effect of any crosstalk originating from the signals (and their corresponding idlers) while propagating in the two directions of the HNLF of the OPC. For these studies, the OPC set-up was slightly modified, allowing only one sub-band (either B1 or B2) to go through the HNLF at a time and thus only generating one conjugated band (either $\mathrm{B} 1 *$ or $\mathrm{B} 2 *$ ). In order to guarantee that the optical power in the transmission system remained the same during all tests, the original signals were transmitted together with their idlers in the second half of the link. This was achieved by adding a $3 \mathrm{~dB}$ coupler at the $7 \mathrm{~dB}$ coupler and combining the original signal at the output of the OPC. In all cases, the crosstalk between signals was less than $-27 \mathrm{~dB}$. Here, the crosstalk was defined as the power difference between the

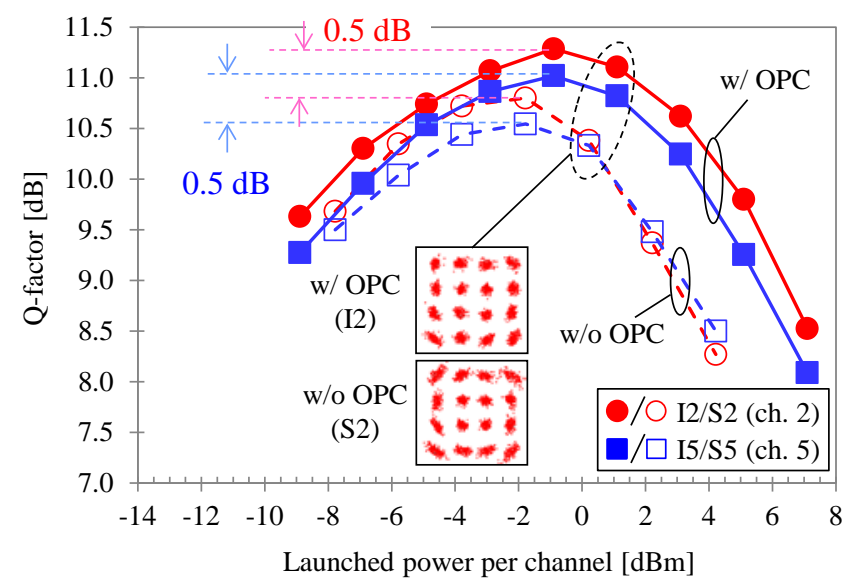

Fig. 5. Q-factors of the middle channels of B1 and B2 (S2 and S5) with and without OPC versus launched power per channel transmitting only B1 and B2 bands.

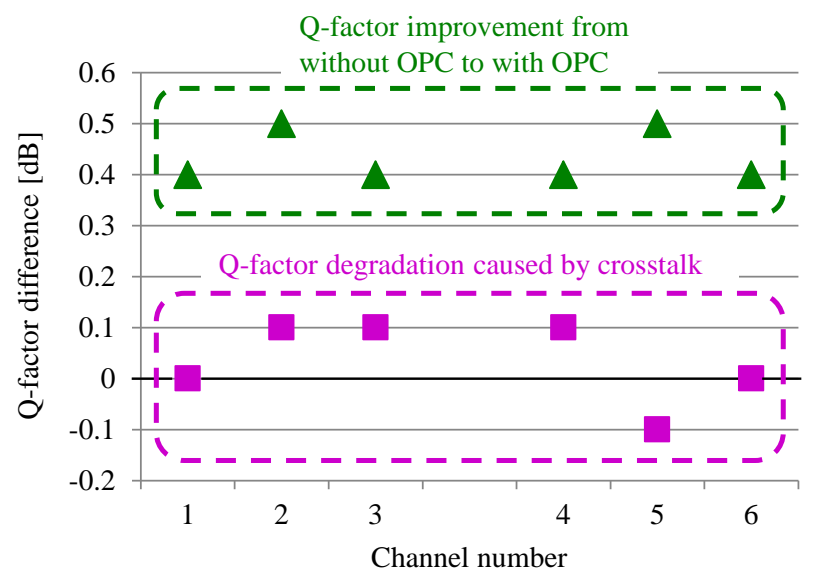

Fig. 6. Q-factor improvement for all the channels from `without OPC’ to `with OPC' and Q-factor difference caused by crosstalk in the HNLF, originating from the channels in each sub-band.

power of the idler and the reflected power when a unidirectional transmission in the HNLF was adopted. The bottom of Fig. 6 (square symbols) shows the difference in Q-factor enhancement caused by this crosstalk, which is within $+/-0.1 \mathrm{~dB}$, indicating that using the nonlinear medium bidirectionally to perform OPC on two separate bands does not sacrifice system performance.

Furthermore, we investigated the Q-factor improvement as the number of WDM channels was increased in the transmission link, i.e. as the nonlinear effects seen by the signals during transmission were increased. Figure 7 shows the received spectrum after $400 \mathrm{~km}$ transmission. The Q-factors of S2 and I2, taken as an example, with and without OPC are shown in Fig. 8. The maximum Q-factor with OPC was $11.0 \mathrm{~dB}$, representing a Q-factor improvement of $0.6 \mathrm{~dB}$. As expected, as the number of WDM channels increased, the optimum launched power per channel was reduced to about $-4 \mathrm{dBm}$ due to the increased nonlinear phase noise. The constellation maps with and without OPC at the launched powers of about -4 and $0 \mathrm{dBm}$ are also reported in Fig. 8. 


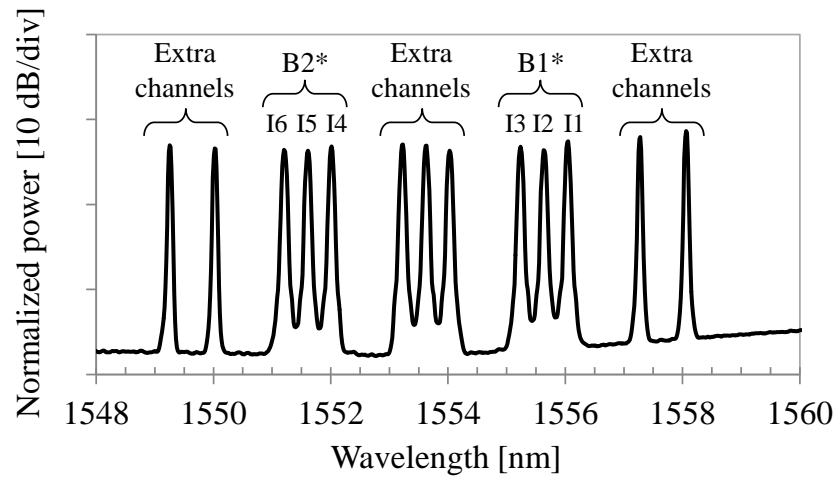

Fig. 7. Received spectrum of idlers including extra WDM channels after 400 km transmission.

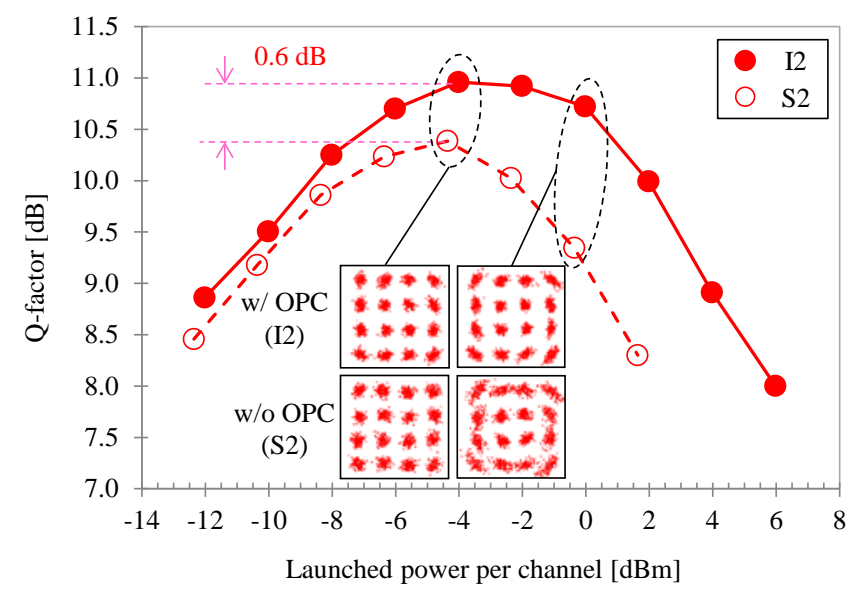

Fig. 8. Q-factors of S2 and I2 versus launched power per channel with extra WDM channels.

\section{TRANSMISSION EXPERIMENT OF 64-QAM}

In the second set of experiments, we studied the transmission of 64-QAM WDM signals over the same $400 \mathrm{~km}$ transmission link with and without OPC. The 64-QAM signals were generated using an arbitrary waveform generator (AWG, Tektronix AWG7122C) with 8 bits resolution, operating at a sampling rate of $10 \mathrm{GSa} / \mathrm{s}$. At the receiver, an EDFA was followed by a $0.4 \mathrm{~nm}$ tunable OBPF, centered at the wavelength of the channel under test, and the signal was detected using a coherent receiver followed by a 4-channel, $16 \mathrm{GHz}, 40 \mathrm{GSa} / \mathrm{s}$ real-time scope. Offline processing was implemented for signal demodulation and BER evaluation. Figure 9 shows a typical received spectrum of the six conjugated copies of the signals after $400 \mathrm{~km}$ transmission when OPC was in place; however, a similar spectrum is obtained without OPC. The corresponding BER curves for the middle channels in each band (S2 and S5) are shown in Fig. 10(a), plotted as a function of launched power per channel into each fiber span. A typical back-to-back BER (corresponding to channel 2), i.e. without the transmission line, is plotted as the reference green line. The BER of our 64-QAM signal $\left(2.7 \times 10^{-2}\right)$ was limited by the bandwidth $(9.6 \mathrm{GHz}$ at -20 $\mathrm{dB}$ ) and effective number of bits of our AWG. Both BER measurements of I2 and I5 (the respective conjugates of S2 and S5) showed improved performance for all launched powers

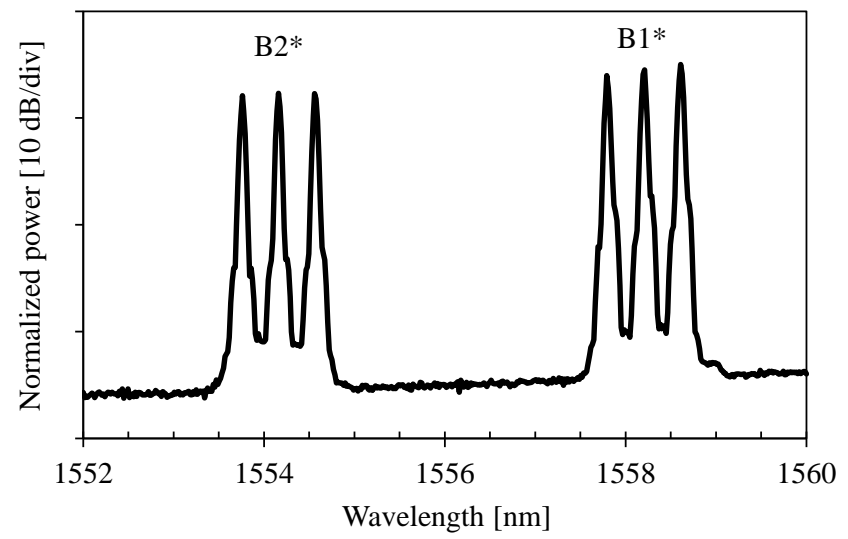

Fig. 9. Example of received spectrum after transmission.

relative to the case without OPC, indicating that the OPC successfully mitigated the nonlinearity-induced transmission impairments. Additionally, the optimum launched power per channel was increased by about $4 \mathrm{~dB}$ for the OPC case due to the fiber Kerr nonlinearity mitigation that it provided. From the BER measurements, we calculated the Q-factor values by using the equation: $\mathrm{Q}=20 \log _{10}\left\{\sqrt{2} \operatorname{erfc}^{-1}(2 \mathrm{BER})\right\}$. Q-factor improvements of about $2.5 \mathrm{~dB}$ and $1.8 \mathrm{~dB}$, respectively, were observed, as shown in Fig. 10(a). Original Q-factors and Q-factor improvements were measured for all remaining channels as shown in Fig. 10(b) and (c), respectively. Although the best Q-factors without OPC were quite low from $1.3 \mathrm{~dB}$ to $1.8 \mathrm{~dB}$ for all channels, the Q-factors with OPC were improved up to $4.3 \mathrm{~dB}$. As a result, a Q-factor improvement of at least 1.2 $\mathrm{dB}$ and up to $2.5 \mathrm{~dB}$ was observed. Again, as was noticed in the previous experiment, the middle channels (which are the ones most affected by nonlinearity), are the ones that can be improved the most through the use of OPC. Furthermore, it is notable that the Q-factor improvement for the 64-QAM WDM signals is significantly better than that previously achieved for the 16-QAM WDM signals (compare Fig. 6 to Fig. 10(b)). This indicates that OPC may have a greater benefit when employed in the transmission of more complex modulation formats that require a higher OSNR, as also demonstrated in [17]. Examples of constellation diagrams with and without OPC at their optimum launched powers, as well as of the back-to-back case for channel 5, are also reported in Fig. 10(c). The reduction of noise through the use of OPC is visible for all symbols by comparing the corresponding constellation diagrams.

\section{CONCLUSIONS}

We have demonstrated an OPC system that does not sacrifice any additional bandwidth during conjugation, and tested its performance by placing it close to the middle of a $400 \mathrm{~km}$ installed transmission fiber link for both 16- and 64-QAM WDM signals. A Q-factor improvement of more than $0.4 \mathrm{~dB}$ was measured for all six 10 Gbaud 16-QAM signals relative to the case when OPC was not used. This value increased to $0.6 \mathrm{~dB}$ when the number of WDM channels was increased to 13 , which indicates that OPC may improve the performance further as the number of WDM channels increases. We also 


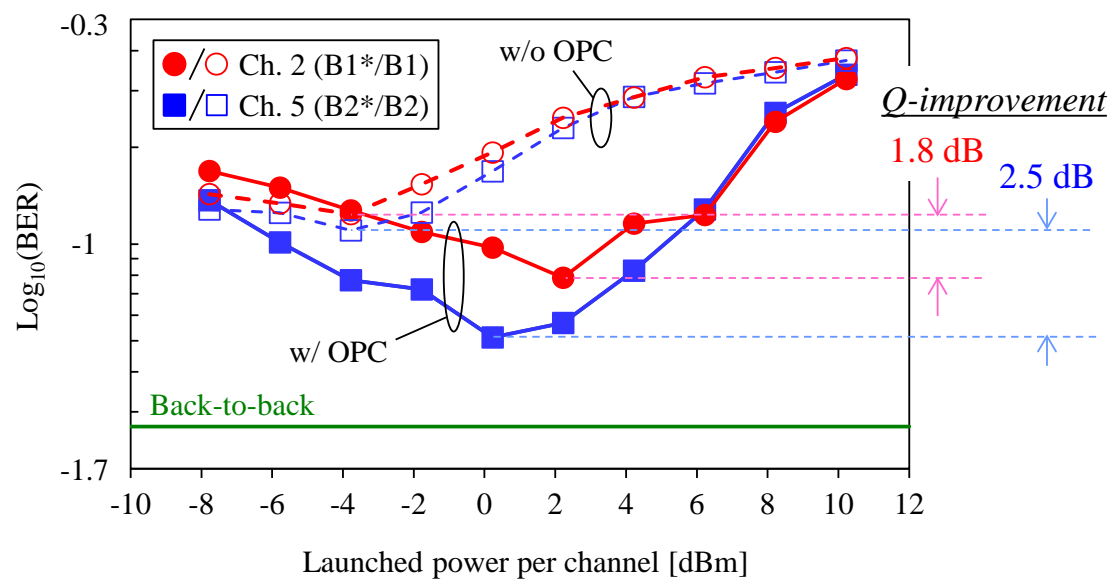

(a)

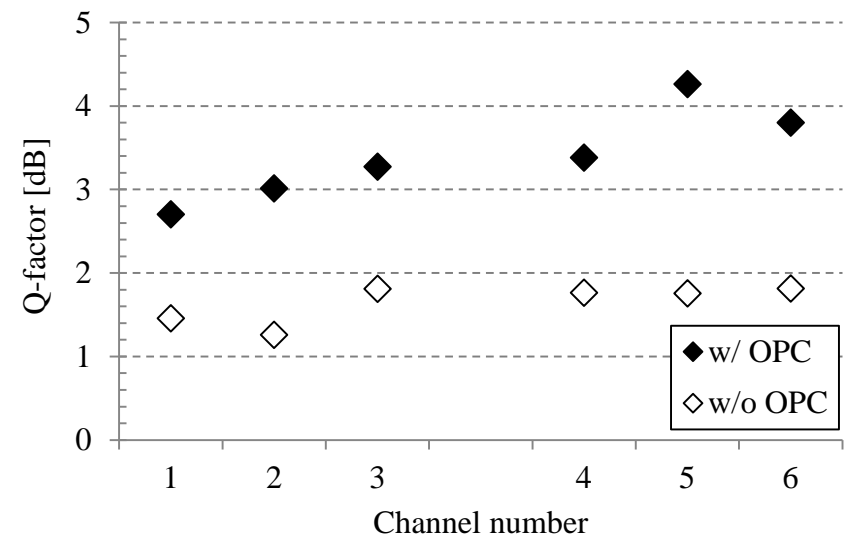

(b)

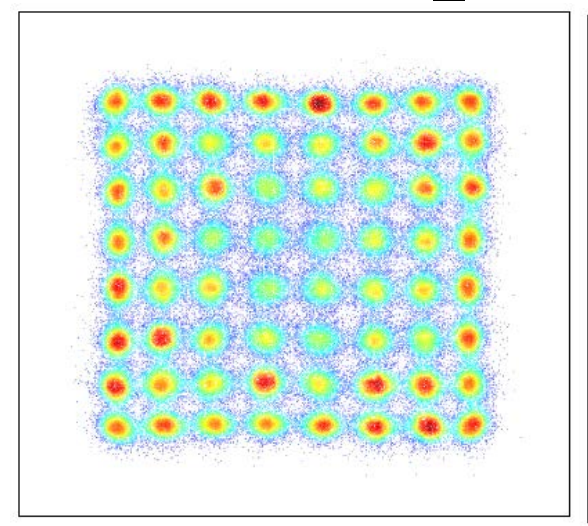

(i) Back-to-back

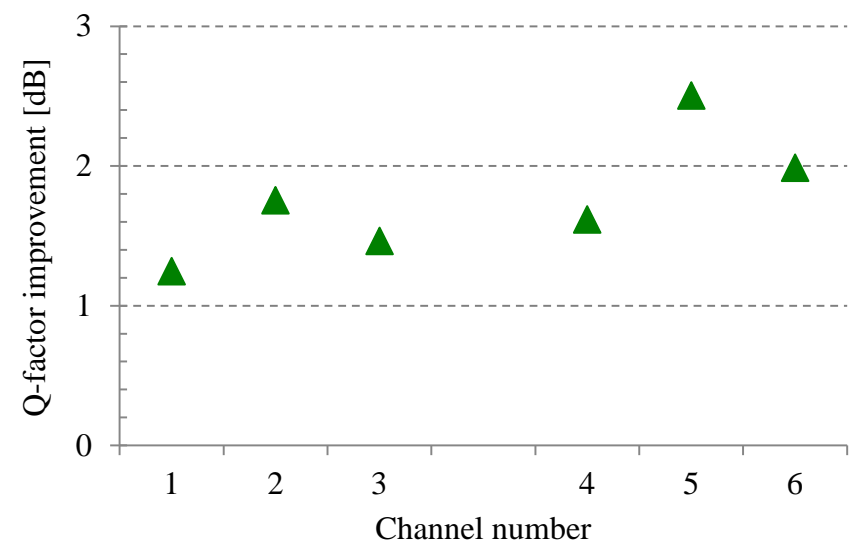

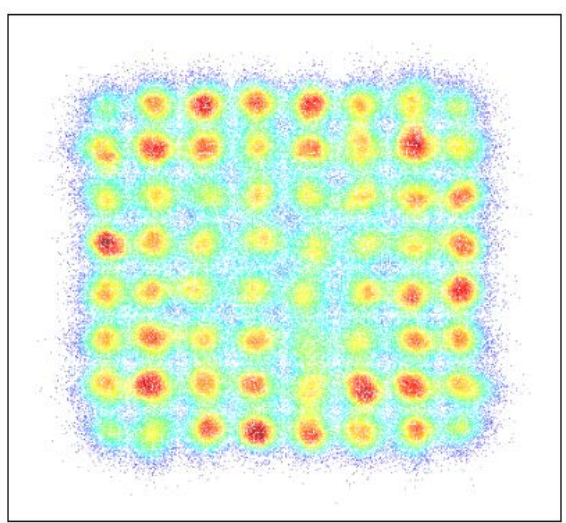

(ii) without OPC (c)

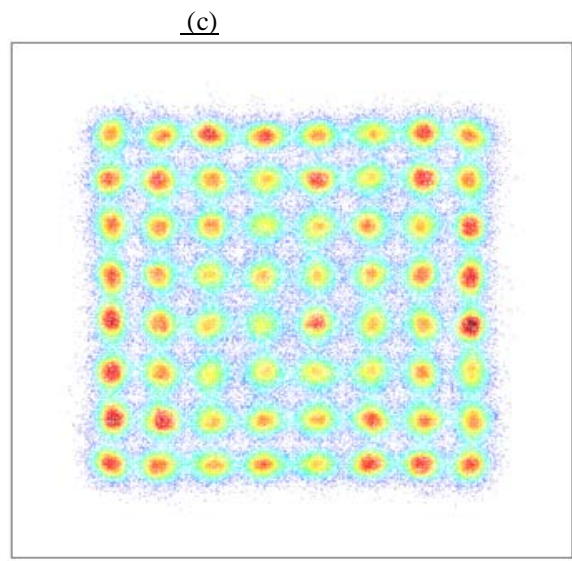

(iii) with OPC

Fig. 10. Experimental results. (a) BER curves for the middle channels of B1 and B2 (S2 and S5) and B1* and B2* (I2 and I5) with and without OPC versus launched power per channel; the back-to-back BER curve for channel 5 is also reported. (b) Original Q-factor and (c) Q-factor improvement for all the six channels from without OPC to with OPC (d) Examples of received constellation diagrams at their optimum launched power for S5/I5 (channel 5) for the back-to-back, without and with OPC case.

experimentally demonstrated fiber nonlinearity mitigation for six 10 Gbaud 64-QAM signals using the same OPC setup and transmission link. Q-factor improvements of up to $2.5 \mathrm{~dB}$ were measured when OPC was in place.

\section{REFERENCES}

[1] T. J. Xia, Glenn A. Wellbrock, M. Huang, S. Zhang, Y. Huang, D. Chang, S. Burtsev, W. Pelouch, E. Zak, H. Pedro, W. Szeto, and H. Fevrier, "Transmission of 400G PM-16QAM channels over long-haul distance with commercial all-distributed Raman amplification system and aged standard SMF in field," presented at the Optical Fiber Communication Conf., San Francisco, CA, USA, Mar. 2014, Paper Tu2B.1.

[2] T. Kobayashi, A. Sano, A. Matsuura, Y. Miyamoto, and K. Ishihara, "Nonlinear tolerant spectrally-efficient transmission using PDM 
64-QAM single carrier FDM with digital pilot-tone,” J. Lightwave Technol., vol. 30, no. 24, pp. 3805-3815, Dec. 2012.

[3] R.-J. Essiambre and R. W. Tkach, "Capacity trends and limits of optical communication networks,” Proc. of the IEEE, vol. 100, no. 5, pp. 10351055, May 2012.

[4] E. Ip, "Nonlinear compensation using backpropagation for polarization-multiplexed transmission,” J. Lightwave Technol., vol. 28, no. 6, pp. 939-951, March 2010.

[5] F. Parmigiani, "Phase sensitive amplifiers and their applications," presented at the Optical Fiber Communications Conf., Los Angeles, CA, USA, Mar. 2015, Paper W4C.1.

[6] S. L. I. Olsson, B. Corcoran, C. Lundström, T. A. Eriksson, M. Karlsson, and P. A. Andrekson, "Phase-sensitive amplified transmission links for improved sensitivity and nonlinearity tolerance", J. Lightwave Technol., vol. 33, no. 3, pp. 710-721, Feb 2015.

[7] Z. Tong, C. Lundström, P. A Andrekson, C. J. McKinstrie, M. Karlsson, D. J. Blessing, E. Tipsuwannakul, B. J. Puttnam, H. Toda, and L Grüner-Nielsen, "Towards ultrasensitive optical links enabled by low-noise phase-sensitive amplifiers,” Nature Photonics, vol. 5, no. 7, pp. 430-436, July 2011.

[8] X. Liu, A. R. Chraplyvy, P. J. Winzer, R. W. Tkach, and S. Chandrasekhar, "Phase-conjugated twin waves for communication beyond the Kerr nonlinearity limit,” Nature Photonics, vol. 7, pp. 560568, July 2013.

[9] K. Solis-Trapala M. Pelusi, H. N. Tan, T. Inoue, and S. Namiki, "Optimized WDM transmission impairment mitigation by multiple phase conjugations,” J. Lightwave Technol., vol. 34, no. 2, pp. 431-440, Jan. 2016

[10] K. Solis-Trapala, T. Inoue, and S. Namiki, "Signal power asymmetry tolerance of an optical phase conjugation-based nonlinear compensation system," presented at the European Conf. Optical Communication, Cannes, France, Sept. 2014, Paper We.2.5.4.

[11] I. Sackey, F. Da Ros, J. Fischer, T. Richter, M. Jazayerifar, C. Peucheret, K. Petermann, and C. Schubert, "Kerr nonlinearity mitigation: Mid-link spectral inversion versus digital backpropagation in 5×28-GBd PDM 16-QAM signal transmission,” J. Lightwave Technol., vol. 33, no. 9, pp. 1821-1807, May 2015.

[12] I. Sackey, F. Da Ros, M. Jazayerifar, T. Richter, C. Meuer, M. Nölle, L. Molle, C. Peucheret, K. Petermann, and C. Schubert, "Kerr nonlinearity mitigation in $5 \times 28$-GBd PDM 16-QAM signal transmission over a dispersion-uncompensated link with backward-pumped distributed Raman amplification,” Optics Express, vol. 22, no. 22, pp. 27381-27391, Nov. 2014.

[13] A. D. Ellis, M. Tan, M .A. Iqbal, M. A. Z. Al Kahteeb, V. Gordienko, G. S Mondaca, S. Fabbri, M. F. C. Stephens, M. E. McCarthy, A. Perentos, I. D. Phillips, D. Lavery, G. Liga, R. Maher, P. Harper, N. J. Doran, S. K. Turitsyn, S. Sygletos, and P. Bayvel, " $4 \mathrm{~Tb} / \mathrm{s}$ transmission reach enhancement using $10 \times 400 \mathrm{~Gb} / \mathrm{s}$ super-channels and polarization insensitive dual band optical phase conjugation,” J. Lightwave Technol., vol. 34, no. 8, pp. 1717-1723, April 2016.

[14] T. Umeki, T. Kazama, H. Ono, Y. Miyamoto, and H. Takenouchi, "Spectrally efficient optical phase conjugation based on complementary spectral inversion for nonlinearity mitigation,” presented at the European Conf. Optical Communication, Valencia, Spain, Sept. 2015, Paper We.2.6.2.

[15] H. Hu, R. M. Jopson, A. H. Gnauck, M. Dinu, S. Chandrasekhar, C. Xie, and S. Randel, "Parametric amplification, wavelength conversion and phase conjugation of a 2.048-Tbit/s WDM PDM 16-QAM signal,” J. Lightwave Technol., vol. 33, no. 7, pp. 1286-1291, April. 2015.

[16] I. D. Phillips, M. Tan, M. F. C. Stephens, M. E. McCarthy, E. Giacoumidis, S. Sygletos, P. Rosa, S. Fabbri, S. T. Le, T. Kanesan, S. K. Turitsyn, N. J. Doran, P. Harper, and A. D. Ellis, "Exceeding the nonlinear-Shannon limit using Raman laser based amplification and optical phase conjugation," presented at the Optical Fiber Communication Conf., San Francisco, CA, USA, Mar. 2014, Paper M3C.1.

[17] K. Solis-Trapala, M. Pelusi, H. N. Tan, T. Inoue, S. Suda, and S. Namiki, "Doubled transmission reach for DP-64QAM signal over field-deployed legacy fiber systems enabled by MSSI," presented at the European Conf. Optical Communication, Valencia, Spain, Sept. 2015, Paper Mo.3.6.2.

[18] A. D. Ellis, M. A. Z. Al Khateeb, and M. E. McCarthy, "Impact of optical phase conjugation on the nonlinear Shannon limit," presented at the Optical Fiber Communication Conf., Anaheim, CA, USA, Mar. 2016, Paper Th4F.2.
[19] S. Namiki, H. N. Tan, K. Solis-Trapala, and T. Inoue, "Signal-transparent wavelength conversion and light-speed back propagation through fiber," presented at the Optical Fiber Communication Conf., Anaheim, CA, USA, Mar. 2016, Paper Th4F.1.

[20] S. Yoshima, Y. Sun, K. R. H. Bottrill, F. Parmigiani, P. Petropoulos, and D. J. Richardson, "Nonlinearity mitigation through optical phase conjugation in a deployed fibre link with full bandwidth utilization," presented at the European Conf. Optical Communication, Valencia, Spain, Sept. 2015, Paper We.2.6.3.

[21] S. Yoshima, Z. Liu, Y. Sun, K. R. H. Bottrill, F. Parmigiani, P. Petropoulos, and D. J. Richardson, "Nonlinearity mitigation for multi-channel 64-QAM signals in a deployed fiber link through optical phase conjugation,” presented at the Optical Fiber Communication Conf., Anaheim, CA, USA, Mar. 2016, Paper Th4F.4.

[22] S. Yan, E. Hugues-Salas, V. J. F. Rancano, Y. Shu, G. M. Saridis, B. R. Rofoee, Y. Yan, A. Peters, S. Jain, T. May-Smith, P. Petropoulos, D. J. Richardson, G. Zervas, and D. Simeonidou, "Archon: a function programmable optical interconnect architecture for transparent intra and inter data center SDM/TDM/WDM networking,” J. Lightwave Technol, vol. 33, no. 8, pp. 1586-1595, April 2015.

[23] V. J. F. Rancano, F. Parmigiani, P. Petropoulos, and D. J. Richardson, "100-GHz grid-aligned multi-channel polarization insensitive black-box wavelength converter,” J. Lightwave Technol, vol. 32, no. 17, pp. 30273035, Sept. 2014.

[24] H. Hu, R. M. Jopson, A. H. Gnauck, D. Pilori, S. Randel, and S. Chandrasekhar, "Fiber nonlinearity compensation by repeated phase conjugation in 2.048-Tbit/s WDM transmission of PDM 16-QAM channels," presented at the Optical Fiber Communication Conf., Anaheim, CA, USA, Mar. 2016, Paper Th4F.3.

[25] K. K. Y. Wong, M. E. Marhic, K. Uesaka, and L. G. Kazovsky, "Polarization-independent one-pump fiber-optical parametric amplifier," Photonics Technol. Letters, vol. 14, no. 11, pp. 1506-1508, Nov. 2002.

[26] S. Radic and C. J. McKinstrie, "Two-pump fiber parametric amplifiers," Opt. Fiber Technol., vol. 9, no. 1, pp. 7-23, Jan. 2003. 